\title{
Editorial
}

\section{Starting to Learn About the Costs of Nosocomial Infections in the New Millennium: Where Do We Go From Here?}

\author{
Victoria J. Fraser, MD
}

In this issue of Infection Control and Hospital Epidemiology, three articles report various aspects of the costs associated with nosocomial infections. Hollenbeak et al. ${ }^{1}$ use four different statistical methods to determine the impact of nonrandom selection on the attributable cost of surgical-site infection (SSI) following coronary artery bypass graft (CABG) surgery. In this study, they analyzed data from 41 cases of deep chest infections following CABG surgery at a Midwestern community medical center and compared them with data from 160 randomly selected uninfected controls. Using the hospital's cost accounting system, they compared the costs of infected and uninfected patients by a $t$ test in a matched and unmatched comparison, by regression analysis, and using Heckman's twostage method. Unmatched comparison demonstrated $\$ 20,012$ in excess cost for the infected patients and after 1:1 matching on age, gender, diabetes, renal insufficiency, and length of surgical procedure, the excess cost for the infected patients was $\$ 19,579$, which was $\$ 433$ lower than the unmatched estimate. Forward stepwise logistic regression was then used to estimate the attributable cost of SSI by controlling for other patient- and process-related risk factors that may affect the cost of care, including severity of illness, duration of surgery, gender, obesity, congestive heart failure, diabetes, reexploration for bleeding, and use of an intra-aortic balloon pump. Use of an intra-aortic balloon pump was the only variable besides SSI that significantly affected the cost of CABG surgery. After controlling for the use of an intra-aortic balloon pump, the authors found that deep chest SSI increased cost by $\$ 19,311$. The authors then used Heckman's two-stage procedure to control for the impact of nonrandom selection, which may affect the cost of SSIs. For example, many of the factors that increase the risk of SSIs may also independently increase the cost of care, such as diabetes, heart failure, severity of illness, renal failure, and duration of surgery. The two-stage method corrects for the possibility of nonrandom selection when assessing cost impact. Using this method, the authors found that the economic impact of deep chest SSI was $\$ 14,211$, approximately $\$ 6,000$ (30\%) lower than the cost estimates defined by the other methods. However, the coefficient on the hazard function was not statistically significant, implying that the cost estimates from the three other methods did not suffer from selection bias. Thus, the cost estimates produced by unmatched comparison, matched comparison, and linear regression are reasonable to use.

This study provides excellent, recent data on the cost of deep chest SSI in a community medical center and thus can be used by infection control specialists, epidemiologists, and administrators to understand the financial impact of SSIs. It also outlines some fairly simple methods that can be used by these same groups to understand what the costs of infections are in their own hospitals. This study was done on one surgical procedure in one hospital during a period of increased infection rates.

What we do not know is whether these costs would be approximately the same during periods of lower infection rates, or whether costs are different for low-frequency endemic infections compared with high-frequency infections or outbreaks. We also do not know how these costs compare with costs in different types of hospitals in different geographic areas. Clearly, additional studies are necessary to determine the variations in cost in different types of hospitals and in different regions. Studies also need to be done to determine how the type of insurance (Medicare, 
Medicaid, health maintenance organization, or no insurance) influences the cost of infections and the actual reimbursement to the hospitals and physicians using real data rather than estimates of what should happen. This study did not examine the costs of superficial chest infections or leg infections, thus we do not have complete information on the relative economic impact of all of these infections.

Whitehouse et al. $^{2}$ performed a pairwise-matched case-control study within a cohort to determine the impact of orthopedic SSI on quality of life, length of stay (LOS), and cost at a tertiary-care university hospital and a community hospital. Procedure type, National Nosocomial Infections Surveillance risk index, age, date of surgery, and surgeon matched cases and controls. Orthopedic SSI increased the initial hospitalization by a median of 1 day and the hospitalization during the follow-up period by a median of 14 days. Patients with SSIs had more hospitalizations (median, 2 vs $1 ; P=.0001$ ) and more surgical procedures (median, 2 vs $1 ; P=.0001$ ). Quality of life was also adversely affected in patients with SSIs. The authors used the SF-36 questionnaire to assess functional status and quality of life 1 year after surgery. Seventy-three (62\%) of the original 118 patients consented to the questionnaire and $46(63 \%)$ could be analyzed in a matched-pair analysis. This study is unique in its use of this questionnaire to assess the impact of infections on functional health status and quality of life. Although the number of matched pairs available for analysis was small, the data suggest significant functional limitations and decreased quality of life in orthopedic patients with SSI.

This study also contributes to the literature by providing recent data on the excess costs associated with orthopedic SSIs. This is an important area and there is little information in the current literature on the economic impact of orthopedic infections. The methods are also easily reproducible and potentially within the scope of many infection control programs. The diversity of the procedures analyzed makes this information useful to centers that do not perform large numbers of any single procedure but provide a diverse spectrum of orthopedic services. To provide specific costs for SSI related to lowfrequency or unusual procedures requires multicenter studies of long duration to collect enough cases and controls. On the down side, the diversity of procedures and the small numbers of individual procedures may dilute the economic estimates and the impact for certain procedures. The most accurate economic estimates will need to be derived from larger studies that are procedure specific and that control for underlying severity of illness and hospital processes that independently impact costs. Unfortunately, cost data were available from the university hospital only, so we cannot compare the costs by hospital type. Larger numbers of patients are also needed for the quality-of-life studies. Future studies should also address functional status and quality of life over time to see how they change in relation to the time of surgery and resolution of the infection.

In the article by Orsi et al., ${ }^{3}$ the increased LOS and cost of nosocomial bloodstream infections (BSIs) are demonstrated in a retrospective cohort study with matching in a large Italian university hospital. Two groups of controls were used: (A) matching on ward, gender, age, diagnosis, central venous catheter, and LOS equal to the interval from admission to infection in a matched case \pm $20 \%$; and (B) matching on the first five and excluding LOS. The attributable mortality rate of nosocomial BSIs was $35.2 \%$ with matching on LOS (group A) and $40.9 \%$ without matching on LOS (risk ratios for death, 2.60 and 3.52, respectively; $P=.0001$ ). The increased LOS was a mean of 19.1 days and a median of 13.0 days with matching on LOS, and a mean of 19.9 days and a median of 15.0 days without matching on LOS. The excess direct cost was Euro 16,356 per case. Of these cases, $79.5 \%$ were primary and $20 \%$ were secondary BSIs. Thirty-three percent of the organisms were coagulase-negative staphylococci, followed by Staphylococcus aureus (19.7\%) and Pseudomonas aeruginosa (11.2\%). Interestingly, the authors noted a decrease in attributable increased LOS when they matched more tightly on duration of hospital stay equal to the interval from admission to infection. The increased hospital stays were $14.6,16.9$, and 26.9 days in the less than $10 \%, 10 \%$ to $20 \%$, and greater than $20 \%$ subgroups, respectively.

This study contributes to the literature by describing the costs of BSIs in an Italian hospital. There are little data on the economic outcomes of nosocomial infections in Europe. It is important to understand regional and geographic differences in the costs of nosocomial infections. Costs of infections should be affected by the type of hospital, the rate of infection, the diagnostic and treatment procedures available, the cost of healthcare delivery, and the payment systems. These variables vary substantially in different parts of the world. The difficulty in measuring the costs of BSIs is further complicated by the difficulty in distinguishing true bacteremias from blood culture contaminants, particularly in the case of coagulase-negative staphylococci. The authors do not clearly address how they dealt with single positive blood cultures of common skin contaminants or how they interpreted coagulase-negative staphylococci, which made up a substantial component of their isolates.

Another important point for this study and all studies of this type is the selection of controls and the variables on which cases are matched. Should controls be matched on underlying severity of illness, factors that clearly impact cost (eg, LOS and procedures), or the most important risk factors for the infection (eg, duration of central venous catheter use in the case of catheter-related BSIs)? Clearly, we need to learn more about the different implications of the matching criteria chosen. Often, characteristics are chosen for matching because they are easily available or clearly documented (eg, age or gender), even if they are not always predictive of costs or infection. Clearly, matching on duration of hospitalization prior to infection is important because it affects cost and risk of infection, but we do not know with certainty how closely we need to match on this variable and additional studies are necessary. 
These articles contribute an additional and important piece of information to our knowledge base on the economic impact of nosocomial infections. Many additional studies are necessary to strengthen our understanding of this complex area. Infection control specialists and epidemiologists will only benefit from partnering with economists and hospital administrators to better understand the financial ramifications of nosocomial infections.

\section{REFERENCES}

1. Hollenbeak CS, Murphy D, Dunagan WC, Fraser VJ. Nonrandom selection and the attributable cost of surgical-site infections. Infect Control Hospital Epidemiol 2002;23:177-182.

2. Whitehouse JD, Friedman ND, Kirkland KB, Richardson WJ, Sexton DJ. The impact of surgical-site infections following orthopedic surgery at a community hospital and a university hospital: adverse quality of life, excess length of stay, and extra cost. Infect Control Hospital Epidemiol 2002;23:183-189.

3. Orsi GB, Di Stefano L, Noah N. Hospital-acquired, laboratory-confirmed bloodstream infection: increased hospital stay and direct costs. Infect Control Hospital Epidemiol 2002;23:190-197. 